\section{Pacific Northwest}

National Laboratory

Operated by Battelle for the

U.S. Department of Energy

\title{
Items Supporting the Hanford Internal Dosimetry Program Implementation of the IMBA Computer Code
}

EH Carbaugh

DE Bihl

January 2008

Prepared for the U.S. Department of Energy under Contract DE-AC05-76RL01830 


\title{
DISCLAIMER
}

This report was prepared as an account of work sponsored by an agency of the United States Government. Neither the United States Government nor any agency thereof, nor Battelle Memorial Institute, nor any of their employees, makes any warranty, express or implied, or assumes any legal liability or responsibility for the accuracy, completeness, or usefulness of any information, apparatus, product, or process disclosed, or represents that its use would not infringe privately owned rights. Reference herein to any specific commercial product, process, or service by trade name, trademark, manufacturer, or otherwise does not necessarily constitute or imply its endorsement, recommendation, or favoring by the United States Government or any agency thereof, or Battelle Memorial Institute. The views and opinions of authors expressed herein do not necessarily state or reflect those of the United States Government or any agency thereof.

\author{
PACIFIC NORTHWEST NATIONAL LABORATORY \\ operated by \\ BATTELLE \\ for the \\ UNITED STATES DEPARTMENT OF ENERGY \\ under Contract DE-AC05-76RL01830
}

Printed in the United States of America
Available to DOE and DOE contractors from the Office of Scientific and Technical Information,
P.O. Box 62, Oak Ridge, TN 37831-0062;
ph: (865) 576-8401
fax: $(865)$ 576-5728
email: reports@adonis.osti.gov

\begin{abstract}
Available to the public from the National Technical Information Service, U.S. Department of Commerce, 5285 Port Royal Rd., Springfield, VA 22161 ph: (800) 553-6847 fax: $(703) 605-6900$ email: orders@ntis.fedworld.gov online ordering: http://www.ntis.gov/ordering.htm
\end{abstract}

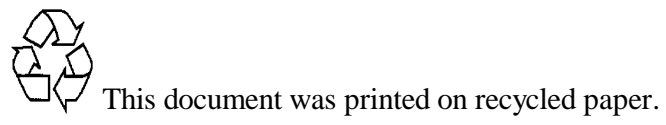




\section{Items Supporting the Hanford Internal Dosimetry Program Implementation of the IMBA Computer Code}

Eugene H. Carbaugh

Don E. Bihl

January 2008

Prepared for the U.S. Department of Energy under Contract DE-AC05-76RL01830 



\title{
Items Supporting the Hanford Internal Dosimetry Program Implementation of the IMBA Computer Code
}

\author{
Eugene H. Carbaugh
}

Don E. Bihl

\section{Summary}

The Hanford Internal Dosimetry Program has adopted the computer code IMBA (Integrated Modules for Bioassay Analysis) as its primary code for bioassay data evaluation and dose assessment using methodologies of ICRP Publications 60, 66, 67, 68, and 78. The adoption of this code was part of the implementation plan for the June 8, 2007 amendments to 10 CFR 835. This information release includes action items unique to IMBA that were required by PNNL quality assurance standards for implementation of safety software. Copies of the IMBA software verification test plan and the outline of the briefing given to new users are also included.

The initial version of the user procedure for the IMBA code has been released as a separate document under information release PNNL-17997 ("Internal Dosimetry Procedure for Use of IMBA Code, PNL-MA-565, 500-11, Rev. 0,” Don E. Bihl, December 2007). 


\section{Hanford Internal Dosimetry Program Actions Related to Adoption of IMBA}




\section{Hanford Internal Dosimetry Actions Related to Adoption of IMBA}

The Hanford Internal Dosimetry Program (HIDP) has moved to adopt the IMBA (Integrated Modules for Bioassay Analysis) computer code as its primary code for bioassay data evaluation and internal dose calculations as part of its implementation of the June 8, 2007 amendment to 10 CFR 835.

The IMBA code is commercially available from the United Kingdom Health Protection Agency (UKHPA), and is internationally recognized as a tested and reliable state-of-the-art internal dosimetry code for the ICRP 60/68 dosimetry system. A key factor in the choice of IMBA has been its inclusion in the DOE Safety Software Central Registry (toolbox), which is defined by DOE Office of Health, Safety, and Security as, "The toolbox codes are compliant with the DOE Safety Software Quality Assurance (SSQA) requirements of DOE O 414.1C and its guidance, DOE G 414.1-4. The toolbox codes are routinely used by DOE to perform calculations and develop data used to establish the safety basis for DOE facilities and their operation, and to support the variety of safety analyses and safety evaluations developed for these facilities. The collection of toolbox codes is referred to as the DOE Safety Software Central Registry."1

The HIDP adoption of IMBA requires several items related to software quality assurance that are addressed in the Safety Software subject area of SBMS and were highlighted to the HIDP Manager by the software QA engineer. These items are addressed by this report.

1. Configuration Management. The HIDP Manager is responsible for configuration management of the HIDP-license copies of IMBA. The HIDP license allows copies of the code to be distributed to five users. The list of authorized users is maintained in the HIDP RIDS under the T7.1 Computer Codes category, Software Log folder. Record copies of IMBA documentation are included in the IMBA folder in the same T7.1 RIDS category. As of this report, the original disk transmittal of IMBA Professional Plus version 4.0.43 was loaded directly onto each of the user's machines. Additional configuration management actions are pending issue of the Radiation and Health Technology Software Configuration Management Plan.

2. Staff Training and Qualification on the Code. Initial training of HIDP staff in the use of the code is documented in their training records, and occurred by briefing by a subject matter expert (Don Bihl) with extensive experience with the code. This training included the qualification of the staff member undergoing training by demonstrating proper execution of bioassay and dose assessment functions of the code. The training method used to qualify IMBA users is consistent with the requirements specified in PNL-MA-870, procedure AP-12, R $\omega H$ H Training Program.

3. Procedure for Code Usage. A procedure covering use of the code is under development and will be included in the Hanford Internal Dosimetry Procedures Manual, PNL-MA-565, as Procedure 500-11. Issue of the procedure development is targeted for late December, 2007. Staff training to the procedure will be by reading.

${ }^{1}$ http://hss.energy.gov/csa/csp/sqa/central registry.htm, (verified 12/20/2007). Copy included as Exhibit 1. 
4. Verification of Software. A plan for software verification testing on individual machines is under development. This testing will be required following initial installation on each machine and with each major upgrade to the code or major change in the operating system.

5. Gap Analysis Review and Risk Identification and Management. HIDP reviewed the DOE gap analysis for IMBA and noted that critical recommendations were either resolved or posed acceptable risks to the HIDP. Details are provided as an appendix to this report.

Based on the successful completion of the steps identified above, the computer code IMBA Professional Plus, version 4.0.43 is accepted for use by the HIDP. Authorized use of the code for HIDP applications is contingent upon successful completion of staff training and software verification on the respective machine.
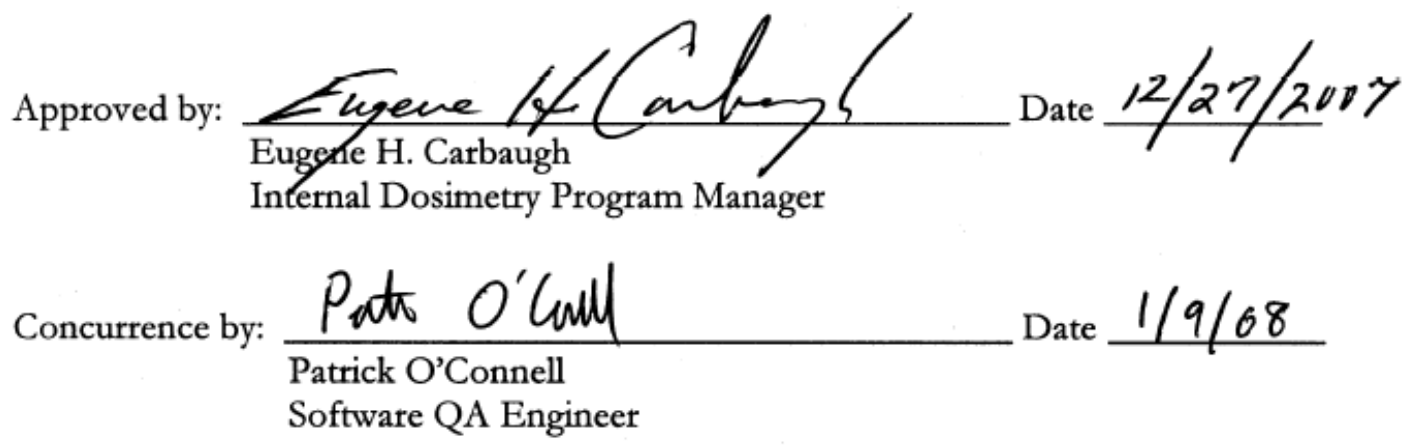


\title{
Appendix
}

\author{
Review of "Gap Analysis for IMBA and DOE Safety Software Central \\ Registry Recommendation, DOE/EH-0711"
}

The Department of Energy (DOE) has issued a final report, "Gap Analysis for IMBA and DOE Safety Software Central Registry Recommendation, DOE/EH-0711, August 2006." The report used as its basis, IMBA Expert ${ }^{\mathrm{TM}}$ USDOE-Edition, version 4.028 and IMBA Professional Plus version 4.0.28. The report noted that those two versions and future minor releases of IMBA Professional Plus versions 4.0.x were recommended for inclusion in the DOE Safety Software Central Registry. That Registry now notes "Upon completion of this evaluation, implementation of critical recommendations, and approval by the DOE, IMBA Expert ${ }^{\mathrm{TM}}$ USDOE-Edition, version 4.028 was included in the DOE Safety Software Central Registry." 2 The citation notes specifically that critical recommendations were implemented, however, documentation of the resolution of the critical recommendations has not been included at the Registry website.

As part of its acceptance process, the HIDP has reviewed the DOE gap analysis report with particular note of its five critical recommendations. This appendix constitutes that review.

The gap analysis identified IMBA as a safety class B software.

CritRec 1. R3-1: Provide IMBA Expert ${ }^{\mathrm{TM}}$ USDOE-Edition V 4.0.28 directly to each licensed DOE user. The recommended distribution method is a CD.

Resolution: This was completed as recommended. The HIDP received a licensed copy of IMBA Professional Plus 4.0.43, which is covered by the recommendations of the report applying to all versions 4.0.x. The procurement of IMBA Professional Plus version 4.0.43 was consistent with the DOE notation that formal support of IMBA Expert ${ }^{\mathrm{TM}}$ USDOE Edition ended December 31, 2006, and that DOE users should upgrade to IMBA Professional Plus. This gap no longer exists.

CritRec 2. R3-2: Create a unique identifier associated with the IPP [IMBA Professional Plus] submodules and implement the use of this identifier throughout development and distribution.

Resolution: This is a recommendation to the code developers and is not pertinent to the HIDP as end user. The current status of the code with regard to this recommendation appears to be resolved so far as the Safety Software Central Registry is concerned. The original concern is considered by the HIDP to be an acceptable risk.

CritRec 3. R3-3: Provide a more obvious and consistent method to confirm that the most recent versions of all sub-modules are being used or downloaded.

${ }^{2}$ http://hss.energy.gov/CSA/CSP/sqa/central registry/IMBA/imba.htm\#top (verified 12/19/2007) Copy included as Exhibit 2. 
Resolution: This is a recommendation to the code developers and is not pertinent to the HIDP as end user. The current status of the code with regard to this recommendation appears to be resolved so far as the Safety Software Central Registry is concerned. The original concern is considered by the HIDP to be an acceptable risk.

CritRec 4. R9-1: Establish and implement an EH problem reporting and notification procedure for IMBA Expert ${ }^{\mathrm{TM}}$ UDSOE-Edition. (Note: This is a recommendation for DOE EH which has now been absorbed into DOE HSS).

Resolution: DOE EH has addressed this by establishing a problem reporting web page ${ }^{3}$. This gap no longer exists.

CritRec 5. R9-2: Implement a formal program with explicit procedures and more accessible records of corrective action activities.

Resolution: This is a recommendation to the code developers (U.K. Health Protection Agency) and is not pertinent to the HIDP as end user. The current status of the code with regard to this recommendation appears to be resolved so far as the Safety Software Central Registry is concerned. The original concern is considered by the HIDP to be an acceptable risk.

${ }^{3}$ http://hss.energy.gov/CSA/CSP/sqa/central registry/IMBA/IMBAreportingform.html (verified 12/21/2007). Copy included as Exhibit 3. 


\title{
Exhibit 1. DOE Safety Software QA Central Registry
}

\author{
http://hss.energy.gov/csa/csp/sqa/central registry.htm (verified 12/20/2007)
}

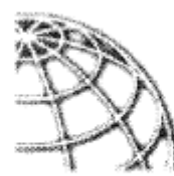
Safety Software Quality Assurance - Central Registry

A significant improvement to the safety software at the Department of Energy (DOE) nuclear facilities was achieved with the development and maintenance of a collection, or "toolbox, "of high-use safety software codes. The toolbox codes are compliant with the DOE Safety Software Quality Assurance (SSQA) requirements of DOE O 414.1C and its guidance, DOE G 414.1-4. The toolbox codes are routinely used by DOE to perform calculations and develop data used to establish the safety basis for DOE facilities and their operation, and to support the variety of safety analyses and safety evaluations developed for these facilities. The collection of toolbox codes is referred to as the DOE Safety Software Central Registry.

The Central Registry currently contains software versions for 7 toolbox codes ALOHA, GFAST, EPICODE, GENII, IMBA, MACCS2, and MELCOR. An additional code, Hotspot, is in the process of implementing recommendations for inclusion into the Central Registry. Prior to inclusion into the Central Registry an evaluation, referred to as a gap analysis, of each code is conducted to identify any "gaps" between the SQA practices and DOE's requirements and criteria for safety software. Code-specific guidance reports were also developed to identify applicable regimes in accident analysis, default inputs, and special conditions for using the toolbox codes for DOE applications. These documents as well as general information are available through the links provided above.

While DOE has established the Central Registry and includes codes into this virtual registry, most were developed outside of DOE (e.g., in the private sector or other Federal agencies). Access to the toolbox codes or their use is subject to agreements, conditions and restrictions established by the code owners or Federal agencies.

The Assistant Secretary for Environment, Safety and Health $(E H-1)$ is responsible for managing the Safety Software Central Registry. However, the toolbox code owners are responsible for ensuring that the codes are maintained in accordance with established requirements. DOE works closely with the code owners to ensure that adequate technical support and training are available.

Although use of the Central Registry toolbox codes is not mandatory, using the codes offer a number of advantages to the DOE and its contractors. These advantages include: 1) the gap analysis evaluation performed provides valuable information as to the quality of the code, 2) the evaluation extends beyond the DOE SSQA criteria to review the code's capability to properly perform safety basis calculations, 3) DOEspecific guidance document that identifies limitations and vulnerabilities not readily found in other code documentation is available, 4) assessments by the field federal and contractor of the toolbox code may be reduced in scope, and 5) continual monitoring and communication to DOE users of new code features and resolutions to defects important to DOE.

DOE also conducted a survey of design codes currently in use across DOE to determine if any should be included in the Safety Software Central Registry. No additional codes were identified for inclusion. A twovolume report (Volume 1; Volume 2) describes the process and identifies the codes evaluated.

For more information on the Central Registry contact Debra Sparkman.

This page was last updated on April 17, 2007 


\title{
Exhibit 2. IMBA Description
}

http://hss.energy.gov/CSACSP/sqa/central registry/IMBA/imba.htm\#top (verified 12/19/2007)

\section{Integrated Modules for Bioassay (IMBA)}

\author{
Current Central Registry Toolbox Version(s): IMBA Expert ${ }^{\mathrm{TM}}$ USDOE Edition version 4.0.28 \\ Code Owner: UK Health Protection Agency (HPA)
}

Description: IMBA Expert ${ }^{\mathrm{TM}}$ (IX) software suite comprises a series of independent modules (referred to as sub-modules) that implement the International Commission on Radiological Protection (ICRP) Publication 66. Human Respiratory Tract Model (HRTM) and the ICRP Publications 30 (series), 67, 68, 69, and 71 biokinetic models.

In 2001, the United Kingdom (UK) National Radiological Protection Board (NRPB), whose functions were absorbed later into the UK Health Protection Agency (HPA), and ACJ \& Associates Inc., began development of an interface (referred to as a shell) for the IMBA modules. This effort was funded in part by the DOE, and in 2003 the first version of IMBA Expert ${ }^{\mathrm{TM}}$ USDOE Edition was distributed to DOE. The final version of IMBA Expert ${ }^{T M}$ USDOE Edition was delivered in 2004. Other, customized versions of IMBA were developed for specific users, including the Canadian power reactor industry and for the National Institute for Occupational Safety and Health. In 2005, IMBA Professional Plus (IPP) was introduced.

Professor Alan Birchall, imba@hpa-rp.org.uk, at UK HPA is the IMBA code architect and program manager. ACJ \& Associates, Inc., controls the distribution of IPP in the United States. IPP updates can be obtained and issues reported at the ACJ \& Associates web site: http: $/ / \mathrm{www}$. acj-associates.com/.

Formal support for IMBA Expert ${ }^{\mathrm{TM}}$ USDOE Edition will cease December 31, 2006. To facilitate continued support of IMBA Expert ${ }^{\text {TM }}$ USDOE Edition for DOE sites, HS-31 has established a problem reporting process. reporting form to report issues and share information related to IMBA Expert ${ }^{\mathrm{TM}}$ USDOE Edition. It is recommended that this site be checked frequently for new information.

In July 2006, the DOE completed an evaluation of IMBA Expert ${ }^{\mathrm{TM}}$ and IMBA Professional Plus (IPP). A gap analysis report was generated that identifies the strengths and weaknesses based upon DOE O 414.1C and DOE G 414.1-4 safety software requirements and criteria. Upon completion of this evaluation, implementation of critical recommendations, and approval by the DOE IMBA Expert ${ }^{T M}$ USDOE Edition version 4.0.28, was included in the DOE Safety Software Central Registry.

Inclusion into DOE's safety software Central Registry provides DOE sites knowledge of the pedigree of the safety software quality assurance (SSQA) level for IMBA Expert ${ }^{\mathrm{TM}}$ USDOE Edition version 4.0.28. If another version of IMBA Expert ${ }^{\mathrm{M}}$ USDOE Edition is used DOE sites must assume the responsibility to determine that the quality assurance level meets DOE SSQA requirements. DOE published recommended guidance for the use of IMBA Expert ${ }^{\mathrm{TM}}$ USDOE Edition version 4.0.28, IPP version 4.0.28, and all future minor releases of IPP 4.0.x.

Issues and concerns related to IMBA:

December 2006 - IMBA Expert ${ }^{\mathrm{TM}}$ USDOE Edition V 4.028 and IMBA Professional Plus V 4.028 have a minor issue associated with the routine that implements the LOD (Level of Detection) fitting protocol. IMBA does not always use the line of best fit below the LOD result if this result is significantly below the rest of the observed data. This is a minor issue. The development organization has reviewed this concern and is evaluating the resolution. It is possible that IMBA's design was intended to prevent users from forcing the line of best fit too far from the majority of the observed data.

\section{Reports and publications related to IMBA:}

HPA IMBA Web Site, http//www.hpa.org uk/radiation/publications/software/imbapro\%5Fplus/.

ACJ \& Associates Inc Web Site, http://www.acj-associates.com/Newsitems/IMBANews.htm 
DOE/EH-0711-IMBA Gap Analysis, Gap Analysis for IMBA and DOE Safety Software Central Registry Recommendation, August 2006.

DOE/EH-0712-IMBA Code Guidance, Guidance on Use of IMBA Software for DOE Safety Applications, Revision 1, December 2006

\section{Obtaining IMBA DOE Expert V 4.0.28}

Formal support for IMBA Expert ${ }^{\mathrm{TM}}$ USDOE Edition will end December 31, 2006. DOE users were issued IMBA Expert ${ }^{\mathrm{TM}}$ USDOE Edition V 4.0.28 CD in August 2006. DOE users may contact the DOE Safety Software Central Registry manager, Debra Sparkman for additional information.

DOE has negotiated with HPA a special one time upgrade to IMBA Professional Plus (IPP) for current DOE users of IMBA Expert ${ }^{\mathrm{TM}}$ USDOE Edition at a heavily discounted price. DOE users can purchase IPP from ACJ \& Associates, Inc. Please complete the order form checking the box "I would like to update from IMBA Expert on page 2.

This page was last updated on February 28, 2007 
Exhibit 3. IMBA Problem Reporting Web Page Form

http://hss.energy gov/CSA/CSP/sqa/central_registry/IMBA/IMBAreportingform.html (verified $12 / 21 / 2007$ ).

IMBA Expert ${ }^{\mathrm{TM}}$ USDOE-Edition, Problem Reporting Web Page Requirements \& Description 

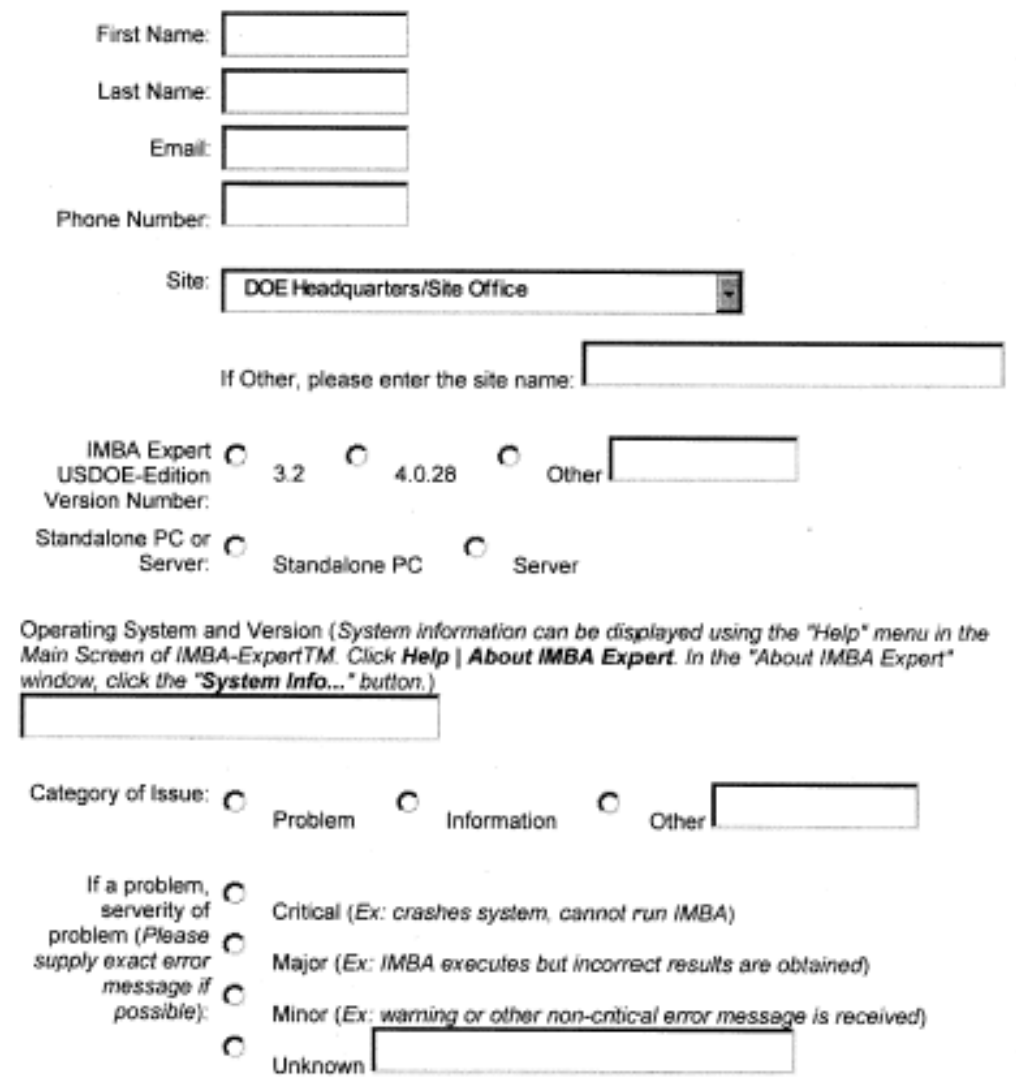

If a problem, description of the problem (Be specific. Include exact emor messages if possibie, steps performed preceding probiem, include the ix file, if possibic, what type of caiculation was being performed)

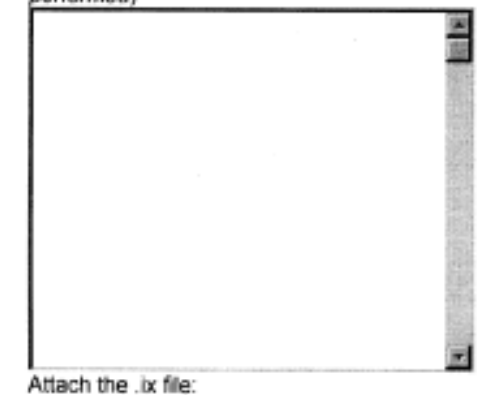

\section{Sutemt Cormem}

Contact Us:

EH-31 Office of Qualty Assurance Programs SQAQEH.DOE.GOV

Exhibit 3, Page 2 of 2

$301-903-6888$ 
Hanford Internal Dosimetry Program IMBA Software Verification Test Plan 


\section{Hanford Internal Dosimetry Program Software Verification Test Plan}

IMBA Professional Plus v. 4.0.43

\section{Computer Make/Model/Number:}

\section{Operating System:}

\section{Reason for testing:}

Tests - Circle the appropriate response.

1. Yes / No Software loads and shows icons for code and supporting documentation.

2. Yes / No Supporting documentation includes files for user manual, appendices A, B, C, and D.

3. Yes / No Code executes upon clicking icon.

For the following tests, it should be noted that IMBA generates results to three or more significant figures, whereas the referenced benchmark, ICRP Publication 78 tabulates results only to two significant figures. The general acceptance criterion of \pm 2 significant figures of the ICRP Publication 78 values allows for some propagation of rounding error.

4. Yes / No Code provides Pu-239 inhalation results consistent within \pm 2 significant figures of those in ICRP Publication 78 for effective dose coefficient and urine, fecal, and in vivo bioassay predictions for days 1, 10 and 180. (Note: the 180-day post acute intake value should be consistent with the ICRP tabulated value for a 360-day monitoring interval, since the monitoring interval assumes a midpoint intake).

Set up parameters: default ICRP model values, 1-Bq acute inhalation intake of Type S material, 5$\mu \mathrm{m}$ AMAD, $f_{1}=1.0 \mathrm{E}-05$. Attach printout of report.

5. Yes / No Code provides Sr-90 inhalation results consistent within \pm 2 significant figures of those in ICRP Publication 78 for effective dose coefficient and urine bioassay predictions for days 1, 10 and 180. (Note: the 180-day post acute intake value should be consistent with the ICRP tabulated value for a 360-day monitoring interval, since the monitoring interval assumes a midpoint intake).

Set up parameters: default ICRP model values, 1-Bq acute inhalation intake of Type F material, 5$\mu \mathrm{m}$ AMAD, $f_{1}=0.3$. Attach printout of report.

6. Yes / No Code provides Cs-137 inhalation results consistent within \pm 2 significant figures of those in ICRP Publication 78 for effective dose coefficient and urine and in vivo (whole body) bioassay predictions for days 1, 10 and 180. (Note: the 180-day post acute intake value should be consistent with the ICRP tabulated value for a 360-day monitoring interval, since the monitoring interval assumes a midpoint intake).

Set up parameters: default ICRP model values, 1-Bq acute inhalation intake of Type F material, 5$\mu \mathrm{m}$ AMAD, $f_{1}=0.99$. (Note: the $f_{1}$ value of 0.99 is a workaround for 1.0. Using 1.0 generates an inconsistent result with day 1 ICRP-78 urine excretion.) Attach printout of report.

7. Yes / No All tests successfully completed and software performs as expected.

Test Performed by: Date

Test Results Reviewed by: Date 
IMBA Verification Test Acceptance Criteria

\section{ICRP 78 Parameter \\ Tabulated \\ Value}

Pu-239 Type S, 5- $\mu$ m AMAD acute inhalation, $f_{1}=1.0 \mathrm{E}-05$

Pu-239 Dose Coefficient $\left(\mathrm{S}_{v} \mathrm{~Bq}^{-1}\right)$ : $\quad$ 8.3E-06

Pu-239 Urine Excretion (Bq per Bq intake)

$\begin{array}{ll}\text { Day } 1 \text { (special) } & 2.3 \mathrm{E}-06 \\ \text { Day } 10 \text { (special) } & 2.3 \mathrm{E}-07\end{array}$

Acute Day 180 (routine day 360) 1.6E-07

Pu-239 Feces Excretion (Bq per Bq intake)

$\begin{array}{ll}\text { Day } 1 \text { (special) } & 1.1 \mathrm{E}-01 \\ \text { Day } 10 \text { (special) } & 6.5 \mathrm{E}-04\end{array}$

Acute Day 180 (routine day 360) 3.7E-05

Pu-239 Lung Retention (Bq per Bq intake)

Day 1 (special) 6.4E-02

Day 10 (special) 5.8E-02

Acute Day 180 (routine day 360) 3.2E-02

\section{Acceptable Range}

Reference
8.1E-06 to $8.5 \mathrm{E}-06$

2.1E-06 to $2.5 \mathrm{E}-06$ $2.1 \mathrm{E}-07$ to $2.5 \mathrm{E}-07$ $1.4 \mathrm{E}-07$ to $1.8 \mathrm{E}-07$

9.0E-02 to $1.3 \mathrm{E}-01$ 6.3E-04 to $6.7 \mathrm{E}-04$ $3.5 \mathrm{E}-05$ to $3.9 \mathrm{E}-05$

6.2E-02 to $6.6 \mathrm{E}-02$ $5.6 \mathrm{E}-02$ to $6.0 \mathrm{E}-02$ $3.0 \mathrm{E}-02$ to $3.4 \mathrm{E}-02$
Table A.12.3

Table A.12.13

Table A.12.13

Table A.12.16

Table A.12.13

Table A.12.13

Table A.12.16

Table A.12.13

Table A.12.13

Table A.12.16

Sr-90 Type F, 5- $\mu \mathrm{m}$ AMAD acute inhalation, $f_{1}=0.3$

Sr-90 Dose Coefficient $\left(\mathrm{Sv} \mathrm{Bq}^{-1}\right)$ : $\quad$ 3.0E-08

Sr-90 Urine Excretion (Bq per Bq intake)

$\begin{array}{ll}\text { Day } 1 \text { (special) } & 6.8 \mathrm{E}-02 \\ \text { Day } 10 \text { (special) } & 4.1 \mathrm{E}-03\end{array}$

Acute Day 180 (routine day 360)
$5.6 \mathrm{E}-05$
2.8E-08 to $3.2 \mathrm{E}-08$

6.6E-02 to $7.0 \mathrm{E}-02$

$3.9 \mathrm{E}-03$ to $4.3 \mathrm{E}-03$

$5.4 \mathrm{E}-05$ to $5.8 \mathrm{E}-05$
Table A.4.3

Table A.4.18

Table A.4.18

Table A.4.20

\section{Cs-137 Type F, 5- $\mu$ m AMAD acute inhalation, ICRP $f_{1}=1.0\left(\right.$ IMBA $\left.f_{1}=0.99\right)$}

Cs-137 Dose Coefficient $\left(\mathrm{Sv} \mathrm{Bq}^{-1}\right)$ :

6.7E-09

Cs-137 Urine Excretion (Bq per Bq intake)

$\begin{array}{ll}\text { Day } 1 \text { (special) } & 7.9 \mathrm{E}-03 \\ \text { Day } 10 \text { (special) } & 2.6 \mathrm{E}-03 \\ \text { (routine day 360) } & 7.0 \mathrm{E}-04\end{array}$

Acute Day 180 (routine day 360)

$7.0 \mathrm{E}-04$

Cs-137 Whole Body Retention (Bq per Bq intake)

Day 1 (special) 6.0E-01

Day 10 (special)

Acute Day 180 (routine day 360)
5.8E-01 to $6.2 \mathrm{E}-01$

3.9E-01 to $4.3 \mathrm{E}-01$

$1.2 \mathrm{E}-01$ to $1.6 \mathrm{E}-01$
Table A.7.3

Table A.7.12

Table A.7.12

Table A.7.14

Table A.7.12

Table A.7.12

Table A.7.14 


\section{Hanford Internal Dosimetry Program IMBA Software User's Briefing}


Training on IMBA Professional Plus

Topics Covered

This training module is intended to be an introduction to the software code IMBA Professional Plus. This training module covers topics not addressed in the procedure on IMBA, which covers more advanced topics.

\section{Main Screen}

\section{Units}

Choice of Date in format $\mathrm{mm} / \mathrm{dd} /$ yyyy or Time in days

"Since" box sets the date from which all days are tracked and plotted. Assumes 00:00 for $\mathrm{hh} / \mathrm{mm}$ if not entered specifically. Usually will use the date of first intake for this box. Choice of units for intake and dose. Note: all doses are 50-yr committed.

\section{Intake Scenarios}

Routes and Modes

Start and end times for chronic intakes; choice of using days or dates. If dates, has to be consistent with the Since date established in the Units section.

Demo of multiple intakes option.

\section{Radionuclides}

How to select Indicator Radionuclide and see decay scheme

How to select Associated Radionuclides - must express as activity \% relative to Indicator Radionuclide.

Associated RN have no relationship to bioassay calculations or calculation of intake; they only come into play in dose calculations.

Example: 20-yr old weapons grade Pu mixture

Indicator $\mathrm{RN}=\mathrm{Pu}-239$

Associated $\mathrm{RN}=\mathrm{Pu}-238$ at $12.67 \%$

$\mathrm{Pu}-240$ at $23.57 \%$

$\mathrm{Pu}-241$ at $545.9 \%$

Am-241 at $28.77 \%$

Note that Associated $\mathrm{RN}$ have same lung type as the Indicator $\mathrm{RN}$, but elements will follow own biokinetic models once they leave the lung. Changes to a biokinetic model only apply to the Indicator RN not the Associated RN, even if both radionuclides are the same element.

Demo uranium mixtures and default specific activities. Note that IMBA specific activities not the same as ID Tech. Basis Table 7-3, even for U nat.

\begin{tabular}{|l|l|l|l|l|l|l|}
\hline & \multicolumn{2}{|c|}{ Unat } & \multicolumn{2}{c|}{ DU } & \multicolumn{2}{c|}{ RU } \\
\hline U-234 & .005367 & .0057 & .001000 & .0005 & .02898 & .0082 \\
\hline U-235 & .7204 & .7204 & .19907 & .2500 & 3.500 & .9700 \\
\hline U-236 & 0.0 & 0.0 & .0003109 & 0.0 & 0.0 & .0680 \\
\hline U-238 & 99.2742 & 99.2739 & 99.7996 & 99.7500 & 96.4722 & 98.9500 \\
\hline $\begin{array}{l}\text { Sp. Act. } \\
\text { (pCi/mg) }\end{array}$ & 683.0 & 705.4 & 402.1 & 371.8 & 2202 & 909.9 \\
\hline
\end{tabular}

* IMBA based on 3.5\% low enrichment; note lack of U-236 which is a mistake.

Model Parameters 
How to load ICRP defaults for each Route type

Under Inhalation, demo each respiratory tract compartment

Deposition: light worker vs. heavy worker breathing rate

AMAD and sigma g calculations

Particle transport: demo rate constants and deposition fractions

Absorption model: demo options and rate constants

Demo GI tract, Bioassay, and Biokinetics buttons

\section{Dose Calculations}

Establish intake

How to choose dose from Indicator RN and/or Associated RN.

How to change radiation weighting factors.

How to change tissue weighting factors.

Note: won't be using the 10CFR835 option now.

Demo difference between Equiv dose and Eff dose. Equiv dose = actual dose to organ;

Eff dose shows doses that contribute to effective dose calculation.

If have Associated RN, have to add doses from Indicator RN and Associated RN to get

total doses. IMBA does not produce total doses when Associated RN are used. (Use

Excel.)

If have chronic intakes or multiple intakes, can get committed doses by year by checking

Annual Committed Dose option.

How to download doses to Excel. (Edit, Copy)

\section{Reports}

Main screen. How to customize reports.

Save and Open will go to JABASOFT \IMBAEXUS \USERDATA \file name.

\section{Bioassay Calculations}

Bioassay to Intake Mode:

How to select type of bioassay. Can select more than one type, all of which are used to determine intake. Open tool and select bioassay type to match type checked in previous section.

How to open a table and enter data.

Blue columns.

Measurement Time - day or date. Can change between using

Tool/change units to days steps on the Bioassay Calculation screen. Measurement date is the date that will be plotted. If collection stretches over 2 days, use the last day; if collection stretches over 3 days (e.g., 24-hr sim.), use the midday.

Collection Period - for urine or feces, use actual fraction of a day if less than a day, e.g. 12-hr sample use 0.5 day. For 24-hr sample or 24-hr sim. use 1 day.

Measurement Rate - for in vivo counts, this is the total activity in the body or organ; for excreta, this is the activity excreted per day. If lab results are for a 12-hr sample, double the results to enter into IMBA. If click on header, can enter the same number down the column.

Data Type -2 choices: 1) < limit of detection, 2) real, 3) exclude (outlier) - shows in plot but is ignored by fitting routine.

Error Distribution - normal or lognormal. Right click and chose.

Measurement Error - column for uncertainties in the measurement data. Can enter actual 1-sigma uncertainties or can manufacturer uncertainties by right clicking on a 
cell in this col. Double uncertainties if have doubled the results to put into

Measurement Rate. Show options for generating uncertainties. K can be any non-zero value if Error Distribution is "normal." Discuss options for $\mathrm{K}$ if Error Distribution is lognormal. Popup window says will overwrite error, click OK.

Can enter 3 types of bioassay data in separate tables. Then check which types to use to calculate intake. Can use all 3 types at once.

Or can use one of the Bio. Quantity tools to produce a graph.

How to build a graph. (Co-60 case on RHT internal drive for demo.) For reports better to copy data to Excel and build table there. Just use copy and paste to move data to Excel. Note: when entering a long list of bioassay data, use the Save button frequently. The OK button does not mean that the bioassay data have been saved.

Can enter data into Excel first, then copy to the Bioassay Quantities table.

Can import data from an ASCII file using the "ffile import" command by right-clicking on the cell or column into which the data go.

Intake to Bioassay mode:

How to set up dates for excretion/retention curve calculations in calculation section.

How to set up dates for excretion/retention curve calculations in table tool.

How to add expectation curve to the graph. 